METZLER FILM LEXIKON 


\section{METZLER FILM LEXIKON}

Herausgegeben von Michael Töteberg

Verlag J.B. Metzler

Stuttgart · Weimar 


\section{INHALT}

Vorwort V
Filme von A-Z 1
Glossar 658
Bibliographie 665
Filmtitel 682
Regisseurinnen und Regisseure 698
Schauspielerinnen und Schauspieler $\quad 703$
Mitarbeiterinnen und Mitarbeiter
Bildquellenverzeichnis $\quad \mathbf{7 3 0}$

Die Deutsche Bibliothek - CIP-Einheitsaufnahme

Metzler-Film-Lexikon / hrsg. von Michael Töteberg. -

Stuttgart ; Weimar : Metzler, 1995

ISBN 978-3-476-00946-3

NE: Töteberg, Michael [Hrsg.]; Film-Lexikon

ISBN 978-3-476-00946-3

ISBN 978-3-476-03496-0 (eBook)

DOI 10.1007/978-3-476-03496-0

Dieses Werk einschließlich aller seiner Teile ist urheberrechtlich geschützt. Jede Verwertung außerhalb der engen Grenzen des Urheberrechtsgesetzes ist ohne Zustimmung des Verlages unzulässig und strafbar. Das gilt insbesondere für Vervielfältigungen, Übersetzungen, Mikroverfilmungen und die Einspeicherung und Verarbeitung in elektronischen Systemen.

(c) 1995 Springer-Verlag GmbH Deutschland Ursprünglich erschienen bei J. B. Metzlersche Verlagsbuchhandlung und Carl Ernst Poeschel Verlag GmbH in Stuttgart 1995 


\section{Vorwort}

45.000 Titel oder noch mehr: Mit jenen Filmlexika, die das Gesamtangebot im Kino, TV und auf Video zu erfassen versuchen, will dieses Buch nicht konkurrieren. Es beschränkt sich auf eine Auswahl von gut 500 Werken. Sie stammen aus verschiedensten Zeiten und Ländern, haben jedoch eines gemeinsam: Sie repräsentieren die Filmkunst in der hundertjährigen Geschichte des Mediums. René Clair, der die Pionierjahre und die Tonfilm-Revolution miterlebte, hat rückblickend bedauert, daß der Begriff "Film" sowohl das Verfahren der bildlichen Reproduktion wie das Ausdrucksmittel meint: Man nenne ja auch nicht alles, was gedruckt werde, Literatur. Die Filme, die in diesem Lexikon behandelt werden, haben allesamt, auch wenn sie für Außenseiter-Positionen stehen, Filmgeschichte gemacht. Sie zeichnen sich durch ästhetische oder technische Innovationen aus, haben Genres begründet oder verändert, bilden das Repertoire einer Kunst, die hierzulande erst spät wissenschaftliche Reputation erlangt hat.

Die getroffene Auswahl, obwohl mit Fachleuten und Freunden immer neu diskutiert, ist naturgemäß angreifbar. Umfragen nach den 100 besten Filmen sind unter Cineasten ein beliebtes Gesellschaftsspiel, doch konnte es hier nicht um eine subjektive Hitliste gehen. Es galt, die großen Regisseure mit charakteristischen Beispielen aus verschiedenen Schaffensund Stilperioden vorzustellen, zugleich aber die kollektiven Hervorbringungen des Studiosystems nicht zu vernachlässigen. Neben künstlerischer Individualität und ästhetischen Standards, wie sie sich in den Zentren kommerzieller Filmproduktion herausgebildet haben, sollten auch gegenläufige Tendenzen präsent sein: Etablierte und verkrustete Strukturen provozierten stets Erneuerungsbewegungen. Genres wie der Western, der Horrorfilm oder das Melodrama, aber auch politische Propagandastreifen oder monumentale Ausstattungsfilme sind ebenso vertreten wie die verschiedenen Spielarten des Autorenfilms. Die Defizite sind dem Herausgeber bewußt: Der Dokumentarfilm, darin spiegelt sich die Kinosituation, ist in diesem Lexikon gewiß unterrepräsentiert.
Der europäische Film und das amerikanische Kino haben ein Übergewicht, während die Kinematographien von Ländern der Dritten Welt nur mit wenigen Werken aufgenommen wurden. Trotzdem hat sich der Herausgeber bemüht, nicht allein den gängigen Kanon nachzuzeichnen. Erfolg, wie er sich an der Kasse oder bei der Oscarverleihung manifestiert, bildete kein entscheidendes Kriterium: Wichtiger erschien die langfristige Wirkung eines Films, wie sie sich in den nachfolgenden Werken oder in der Veränderung von Wahrnehmungsstrukturen beim $\mathrm{Zu}$ schauer zeigt. Der einzelne Film wird stets im Kontext analysiert: innerhalb der Filmgeschichte, mit Bezug auf andere Werke desselben Regisseurs, auf vorhergegangene, parallel entstandene oder - ein Aspekt produktiver Rezeption - von ihm inspirierte Filme, und auch im Zusammenhang mit den ökonomischen und gesellschaftlichen Rahmenbedingungen. Der Film war nie reine Kunst, sondern immer auch das Produkt der Unterhaltungsindustrie seiner Zeit. Produzenten und Verleihfirmen haben aus kommerziellen Motiven, politische Instanzen mit Blick auf die Massenwirkung direkt oder indirekt Einfluß genommen. Profitdenken wie Zensurbehörden sind gleichermaßen verantwortlich dafür, daß berühmte Klassiker der Filmgeschichte nur in verstümmelter oder tendenziös veränderter Fassung in die Kinos kamen.

Das Metzler Film Lexikon versteht sich als Nachschlagewerk für Kinogänger, die sich über Thema und Stil informieren wollen, ohne mit bloßen Inhaltsangaben und fragwürdigen Wertungen zufrieden zu sein. Produktionsgeschichte, Mise-en-scène, Darstellungsstil, Erzählstruktur und Dramaturgie, Kameraführung und Lichtgestaltung, Montage und Musik, die Rezeption, wozu auch Hinweise auf Remakes und Sequels gehören: Dies sind einige Aspekte, auf die in den Artikeln zu den Filmen Wert gelegt wurde. Ein standardisierter Aufbau der Artikel erschien dem Herausgeber nicht sinnvoll. Angeordnet ist das Lexikon nach Originaltiteln, gegebenenfalls erfolgt beim deutschen Verleihtitel ein entspre- 
chender Verweis. Im Kopf der Filmographie werden genannt: das Land, die Produktionsfirma und das Entstehungsjahr, das vom Jahr der Uraufführung abweichen kann; es folgen Längen- und Formatangaben, wobei wiederum das Original, nicht eine eventuelle spätere Bearbeitung (z.B. das spätere Aufblasen von $16 \mathrm{~mm}$ auf $35 \mathrm{~mm}$ oder nachträglich vom Produzenten vorgenommene Kürzungen) entscheidend waren. Bei den Stummfilmen erfolgt die Längenangabe in Metern. Nach den Angaben zu Regisseur (R) und Drehbuchautor (B), gegebenenfalls ergänzt um den Hinweis auf eine literarische Vorlage, folgen Kamera (K), Ausstattung (A), Bauten (Ba), Schnitt (S) und Musik (M). Bei den Darstellern war Beschränkung geboten auf die wichtigsten Hauptdarsteller, in Klammern die Rollennamen.

Die Bibliographien am Schluß des Artikels geben Hinweise auf weiterführende Literatur. Zuerst werden Textausgaben genannt, wobei eine grobe Kategorisierung versucht wurde: Drehbücher können vom realisierten Film abweichen. Filmprotokolle sind nachträglich erstellte Beschreibungen, die wissenschaftlichen Maßstäben genügen; die in den USA üblichen Final Shooting Scripts wurden zu den Filmprotokollen gerechnet. Alle sonstigen Zwischenformen werden Filmtext genannt: In der Regel handelt es sich um die Wiedergabe von Dialogen und knappen Szenenbeschreibungen, jedoch ohne Angaben zu Einstellungen. (Den Verlagsangaben ist, da sich eine "Filmnovelle" leichter verkaufen läßt als eine erste literarische Drehbuchfassung, in den seltensten Fällen zu trauen; auf die Aufnahme von "Büchern zum Film", d.h. in der Regel von Ghostwritern erstellten Romanfassungen, wurde verzichtet.) Enthält der Band zusätzlich Exposés, Treatments, Aufsätze oder Interviews, so wird dies unter dem Obergriff "Materialien" notiert. Die Angaben zur Sekundärliteratur bemühen sich um Benutzerfreundlichkeit: Liegt ein vergriffenes Buch in einer Neuausgabe vor, ist eine Filmkritik in einem Sammelband leichter greifbar, wurde diesen Angaben der Vorzug gegeben. Sigel wurden grundsätzlich aufgelöst, bei der fremdsprachigen Sekundärliteratur wurde englischen und französischen Texten der Vorzug gege- ben. Im Anhang des Lexikons listet eine Bibliographie die wichtigste Standardliteratur zum Thema Film auf; Monographien zu Regisseuren, die mit fünf oder mehr Werken vertreten sind, sind dort gesondert nachgewiesen.

Als Fachberater standen dem Herausgeber Günter Agde, Rolf Aurich, Hans-Michael Bock und Rüdiger Koschnitzki zur Seite. Ohne die Unterstützung von CineGraph (Hamburg), der Stiftung Deutsche Kinemathek (Berlin) sowie dem Deutschen Institut für Filmkunde und dem Deutschen Filmmuseum (beide Frankfurt a.M.) wäre die Literatur-Recherche kaum erfolgreich zu bewerkstelligen gewesen; geholfen haben neben vielen anderen Harald Kern und Philip Roeder. Die Erarbeitung der Bibliographien besorgte der Herausgeber gemeinsam mit Ingo Fließ, der auch das Glossar verfaßte. Ein spezieller Dank geht an Christiane Bornemann und Ulrike Theilig.

Stand und Entwicklung der Technik haben von Beginn an die Filmkunst geprägt. Nach hundert Jahren steht erneut ein Umbruch bevor. Eine Epoche geht zu Ende, eine neue beginnt. Der Herausgeber des Lexikons, dessen Perspektive retrospektiv sein muß, teilt die von Wim Wenders formulierte Zuversicht: "Vor dem Eintritt in das Zeitalter des digitalen elektronischen Bildes ist das Kino vielleicht noch einmal fähig, alle seine Kräfte zusammenzunehmen und das zu tun, wozu es erfunden wurde: dem Menschen des zwanzigsten Jahrhunderts sein eigenes Bild vorzuhalten, sowohl das seiner Realität als auch das seiner Träume.“

Michael Töteberg 